\title{
Evaluation of the effect of neoadjuvant treatment in pancreatic ductal adenocarcinoma
}

\author{
$\underline{\text { Irene ESPOSITO* }}^{*}$
}

Institute of Pathology, University Hospital of Dusseldorf, Dusseldorf, Germany

Lecture: Neoadjuvant treatment (NAT) is currently performed in borderline-resectable and locally advanced, primarily non-resectable pancreatic ductal adenocarcinoma (PDAC). In addition, NAT is advocated by some in resectable PDAC as well. Down-staging and increase of R0-resection rates belong to the major clinical read-outs for NAT in PDAC. There is therefore a strong need for a highly standardized method for pathological examination of resection specimens after NAT in order to provide reliable, comparable results across studies and institutions worldwide.

According to the 8th edition of the AJCC/UICC staging classifications, size is the most important factor to assess the T-category. However, assessment of residual tumor size after NAT represents a major hurdle both by imaging and by pathological examination and substantial evidence for the currently proposed methods is still lacking. In addition, numerous tumor regression grade systems have been proposed in the last years, but they are often scarcely reproducible and/or lack correlation with patients' survival. Recent studies have shown that changes induced by NAT in PDAC, such as fibrosis, cytoplasmic vacuolization and necrosis might also be present in therapy-naïve PDAC, rendering qualitative interpretation difficult. In particular, it seems impossible to distinguish tumor-associated desmoplasia from therapy-induced fibrosis.

Numerous international initiatives, such as the International Collaboration on Cancer Reporting, the International Study Group of Pancreatic Pathologists and the Neoadjuvant Therapy Working Group of the Pancreatobiliary Pathology Society are currently working to provide pathologists all over the world with an easy, highly reproducible, standardized and clinically relevant system to evaluate PDAC after NAT. 